\title{
IDEALS OF COMPACT OPERATORS WITH NAKANO TYPE NORMS IN A HILBERT SPACE
}

\author{
MiCHAEL GIL'
}

Abstract. Let $H$ be a separable Hilbert space with a norm $\|.\|_{H}$. For a compact linear operator $A$ acting in $H$, let $\lambda_{k}(A)$ be the eigenvalues, $s_{k}(A)(k=1,2, \ldots)$ singular values and $\|A\|_{H}=$ $\sup _{x \in H}\|A x\|_{H} /\|x\|_{H}$. Let $\pi=\left\{p_{k}\right\}_{k=1}^{\infty}$ be a nondecreasing sequence of numbers $p_{k} \geqslant 1$. Put

$$
\gamma_{\pi}(A):=\sum_{j=1}^{\infty} \frac{s_{j}^{p_{j}}(A)}{p_{j}} .
$$

We investigate the ideal $X_{\pi}$ of operators satisfying $\gamma_{\pi}(t A)<\infty$ for all $t>0$. In particular, it is proved that for any $A \in X_{\pi}$ we have

$$
\sum_{k=1}^{\infty} \frac{\left|\lambda_{k}(A)\right|^{p_{k}}}{p_{k} v_{A}^{p_{k}}} \leqslant \gamma_{\pi}\left(A / v_{A}\right),
$$

where $v_{A}=\|A\|_{H}$ if $\|A\|_{H}>1$ and $v_{A}=1$ if $\|A\|_{H} \leqslant 1$.

Mathematics subject classification (2010): 47B10, 47A10, 47A11.

Keywords and phrases: Hilbert space, compact operators, estimates for eigenvalues.

\section{REFERENCES}

[1] B. Aqzzouz And R. Nouira, The order ideal of AM-compact operators, Proc. Am. Math. Soc. 134, 12 (2006), 3515-3523.

[2] G. ARsu, On Schatten-von Neumann class properties of pseudodifferential operators. The CordesKato method, J. Oper. Theory 59, 1 (2008), 81-114.

[3] P. ChaisuriYa, AND S. C. ONG, Schatten's theorems on functionally defined Schur algebras, Int. J. Math. Math. Sci. 2005, 14 (2005), 2175-2193.

[4] C. Conde, Geometric interpolation in p-Schatten class, J. Math. Anal. Appl. 340, 2 (2008), 920-931.

[5] I. Doust, AND T.A. GILlESPIE, Schur multiplier projections on the von Neumann-Schatten classes, J. Oper. Theory 53, 2 (2005), 251-272.

[6] N. Dunford, And J. T. SchwartZ, Linear Operators, part II. Spectral Theory, Interscience Publishers, New York, London, 1963.

[7] L. G. Gheorghe, Hankel operators in Schatten ideals, Ann. Mat. Pura Appl., IV Ser., 180, 2 (2001), 203-210.

[8] M.I. GIL', Lower bounds for eigenvalues of Schatten-von Neumann operators, J. of Inequalities in Pure and Appl. Mathem. 8, 3 (2007), Article 66, 7 pp.

[9] M.I. GIL', Inequalities of te Carleman Type For Neumann-Schatten Operator, Asian-European J. of Math. 1, 2 (2008), 203-212.

[10] I. Gohberg, S. Goldberg, And N. Krupnik, Traces and Determinants of Linear Operators, Birkhäuser Verlag, Basel, 2000.

[11] I. C. Gohberg AND M. G. KReIn, Introduction to the Theory of Linear Nonselfadjoint Operators, Trans. Mathem. Monographs, vol. 18, Amer. Math. Soc., Providence, R. I., 1969.

[12] I. C. Gohberg, AND M. G. Krein, Theory and Applications of Volterra Operators in Hilbert Space, Trans. Mathem. Monographs, vol. 24, Amer. Math. Soc., R. I., 1970. 
[13] M. HANSMAnN, Estimating eigenvalue moments via Schatten norm bounds on semigroup differences, Math. Phys. Anal. Geom. 10, 3 (2007), 261-270.

[14] T. KÜHN, AND M. MASTYŁO, Products of operator ideals and extensions of Schatten classes, Math. Nachr. 283, 6 (2010), 891-901.

[15] C. LE MERdy, E. RICARD AND J. Roydor, Completely 1-complemented subspaces of Schatten spaces, Trans. Am. Math. Soc. 361, 2 (2009), 849-887.

[16] J. Lindenstrauss, And L. Tzafriri, Classical Banach Spaces I. Sequence Spaces, Springer, Berlin, 1977.

[17] S. MECHERI, Another version of Anderson's inequality in the ideal of all compact operators, JIPAM, J. Inequal. Pure Appl. Math. 6, 3 (2005), Paper No. 90, 7 p., electronic only.

[18] S. MECHERI, On the orthogonality in von Neumann-Schatten class, Int. J. Appl. Math. 8, 4 (2002), $441-447$.

[19] H. NAKAno, Modulared Semi-ordered Linear Spaces, Tokyo Math. Book Series, I, Tokyo, 1950.

[20] M. SigG, A Minkowski-type inequality for the Schatten norm, J. Inequal. Pure Appl. Math. 6, 3 (2005), Paper No. 87, 7 p.

[21] M. SUNDHÄLL, Schatten-von Neumann properties of bilinear Hankel forms of higher weights, Math. Scand. 98, 2 (2006), 283-319.

[22] M.W. Wong, Schatten-von Neumann norms of localization operators, Arch. Inequal. Appl. 2, 4 (2004), 391-396.

[23] J. XIA, On the Schatten class membership of Hankel operators on the unit ball, Ill. J. Math. 46, 3 (2002), 913-928. 